\title{
Article
}

\section{Pupillometric and saccadic measures of affective and executive processing in anxiety}

Hepsomali, Piril, Hadwin, Julie, Liversedge, Simon Paul and Garner, Matthew

Available at http://clok.uclan.ac.uk/22329/

Hepsomali, Piril, Hadwin, Julie, Liversedge, Simon Paul ORCID: 0000-00028579-8546 and Garner, Matthew (2017) Pupillometric and saccadic measures of affective and executive processing in anxiety. Biological Psychology, 127 . pp. 173-179. ISSN 0301-0511

It is advisable to refer to the publisher's version if you intend to cite from the work. http://dx.doi.org/10.1016/j.biopsycho.2017.05.013

For more information about UCLan's research in this area go to http://www.uclan.ac.uk/researchgroups/ and search for < name of research Group>.

For information about Research generally at UCLan please go to http://www.uclan.ac.uk/research/

All outputs in CLoK are protected by Intellectual Property Rights law, including Copyright law. Copyright, IPR and Moral Rights for the works on this site are retained by the individual authors and/or other copyright owners. Terms and conditions for use of this material are defined in the policies page.

\section{CLoK}

Central Lancashire online Knowledge www.clok.uclan.ac.uk

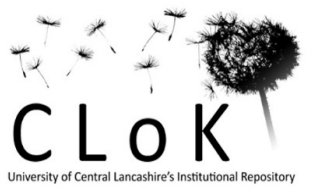




\section{Accepted Manuscript}

Title: Pupillometric and Saccadic Measures of Affective and Executive Processing in Anxiety

Authors: Piril Hepsomali, Julie A. Hadwin, Simon P. Liversedge, Matthew Garner

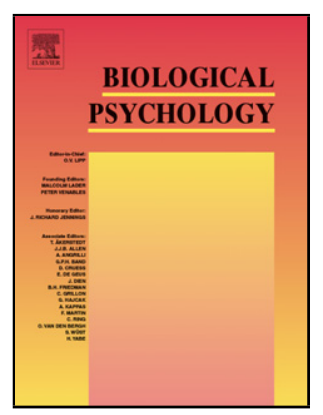

PII: S0301-0511(17)30112-6

DOI: http://dx.doi.org/doi:10.1016/j.biopsycho.2017.05.013

Reference: BIOPSY 7382

To appear in:

Received date: $\quad$ 11-7-2016

Revised date: 22-3-2017

Accepted date: $\quad$ 23-5-2017

Please cite this article as: Hepsomali, Piril, Hadwin, Julie A., Liversedge, Simon P., Garner, Matthew, Pupillometric and Saccadic Measures of Affective and Executive Processing in Anxiety.Biological Psychology http://dx.doi.org/10.1016/j.biopsycho.2017.05.013

This is a PDF file of an unedited manuscript that has been accepted for publication. As a service to our customers we are providing this early version of the manuscript. The manuscript will undergo copyediting, typesetting, and review of the resulting proof before it is published in its final form. Please note that during the production process errors may be discovered which could affect the content, and all legal disclaimers that apply to the journal pertain. 


\section{PUPILLARY AND SACCADIC RESPONSES IN ANXIETY}

$<$ AT $>$ Pupillometric and Saccadic Measures of Affective and Executive Processing in Anxiety $<$ AU $>$ Piril Hepsomali ${ }^{\mathrm{a}^{*}}$ \#\#Email\#\#P.Hepsomali@soton.ac.uk \#\#/Email\#\# ., Julie A. Hadwin ${ }^{a}$, Simon P. Liversedge ${ }^{a}$, \& Matthew Garnera,

$<\mathrm{AFF}>$ a Department of Psychology

$<\mathrm{AFF}>{ }^{\mathrm{b}} \mathrm{Clinical}$ and Experimental Sciences

Highfield

University of Southampton

Southampton SO17 1BJ

UK

\section{Author Note}

This research was supported by funds from an Economic and Social Research Council Doctoral Training Centre studentship (awarded to Piril Hepsomali) and the Department of Psychology, University of Southampton.

$<$ ABS-HEAD $>$ Highlights Anxious individuals produce elevated and longer pupil responses to emotional faces. Elevated pupil responses in anxious individuals are insensitive to changes in task demand (long vs. short delay), conversely low anxious individuals' pupil responses vary with task demand and show evidence of improved processing efficiency during low load. Anxious individuals subsequently make more eyemovement errors in an oculomotor delay task, particularly when task demands are high (i.e. following a long delay).

$$
<\text { ABS-HEAD }>\text { Abstract }
$$

$<$ ABS-P $>$ Anxious individuals report hyper-arousal and sensitivity to environmental stimuli, difficulties concentrating, performing tasks efficiently and inhibiting unwanted thoughts and distraction. We used pupillometry and eye-movement measures to compare high vs. low anxious individuals hyper-reactivity to emotional stimuli (facial expressions) and subsequent attentional biases in a memory-guided pro- and antisaccade task during conditions of low and high cognitive load (short vs. long delay). High anxious individuals produced larger and slower pupillary responses to face stimuli, and more erroneous eye-movements, particularly following long delay. Low anxious individuals' pupillary responses were sensitive to task demand (reduced during short delay), whereas high anxious individuals' were not. These findings provide evidence in anxiety of enhanced, sustained and inflexible patterns of pupil responding during affective stimulus processing and cognitive load that precede deficits in task performance.

$<$ KWD $>$ Keywords: pupillary responses; saccades; anxiety; emotion; effort

\section{Pupillometric and Saccadic Measures of Affective and Executive Processing in Anxiety}

Anxiety is characterised by hyperactivity in physiological, cognitive and behavioural mechanisms in anticipation of threat and in response to threat cues (Bar-Haim, Lamy, Pergamin, Bakermans-Kranenburg, \& van IJzendoorn, 2007). Neuropsychological models of anxiety highlight maladaptive biases in threat appraisal and attention in the aetiology and 


\section{PUPILLARY AND SACCADIC RESPONSES IN ANXIETY}

maintenance of core symptoms that include distractibility, poor concentration, nervous apprehension and worry (Behar, DiMarco, Hekler, Mohlman, \& Staples, 2009; Grupe \& Nitschke, 2013; Mogg \& Bradley, 2016; Sylvester et al., 2012). Hyperactivity in bottom-up stimulus-driven mechanisms (i.e. increased amygdala activation) increase threat appraisal, autonomic arousal, and attentional bias to threat (Bishop, 2009). In addition hypo-activity in goal-directed control processes (i.e., reduced activity in the dorsolateral prefrontal cortex) reduce attentional control and exacerbate attentional biases to threat distractors, particularly when task demands are high (Bishop, 2009). Recent methodological advances provide more sensitive indices of attention and related processes (e.g., pupillometry and eye-movement measures) to examine anxiety-related biases in emotional reactivity to emotional stimuli, and the impact of low and high cognitive load on attention biases and attentional control more broadly.

Pupillary responses of the human eye are modulated by affective and executive processes via central and peripheral sympathetic and parasympathetic divisions of the nervous system (see Andreassi, 2000, for a review). Pupil diameter increases in response to emotional stimuli (both visual and auditory) and correlates with subjective ratings of emotional arousal and autonomic skin-conductance responses (Bradley, Miccoli, Escrig, \& Lang, 2008; Partala \& Surakka, 2003; Rosa, Esteves, \& Arriaga, 2015). Recent evidence suggests that pupillary responses are sensitive to individual differences in threat processing, as reflected in increased pupil responses to angry faces in anxious individuals (Kret, Stekelenburg, Roelofs, \& de Gelder, 2013), fearful faces in anxious youths (Price et al., 2013) and to angry faces in children of anxious mothers (Burkhouse, Siegle, \& Gibb, 2014).

Behavioural studies provide evidence of visuo-spatial biases in selective attention to threat in anxiety. For example, individuals with clinical and sub-clinical anxiety make speeded reaction times (RTs) to visual targets that appear in the spatial location of threat stimuli (e.g. negative pictures and facial expressions; review by Bar-Haim et al., 2007). Likewise eyetracking methods reveal preferential and faster eye-movements towards threat cues in anxiety (Chen, Clarke, Watson, Macleod, \& Guastella, 2014; Mogg, Garner, \& Bradley, 2007). Biases in selective attention have been observed in a range of anxiety groups, including generalized anxiety, social phobia, specific phobias and high trait anxious 'sub-clinical' populations (review by Bar-Haim et al., 2007) and are exacerbated in individuals who concurrently report poor attentional control (Derryberry \& Reed, 2002). Convergent evidence of visuospatial biases for threat in anxiety is consistent with models that propose 'hypervigilance' for threat increases distractibility and interrupts executive processes required to monitor and achieve performance goals (review by Richards, Benson, Donnelly, \& Hadwin, 2014).

Attentional control theory (Eysenck \& Derakshan, 2011; Eysenck, Derakshan, Santos, \& Calvo, 2007) and related models (e.g. Processing Efficiency Theory; Eysenck \& Calvo, 1992) further propose that anxiety reduces executive attention control resources that are required to maintain goal- focus and inhibit task-irrelevant (negative) distractors. These models predict that anxious individuals recruit more resources and increase effort to complete tasks effectively. Consequently, anxiety is thought to be associated with substantial impairment in processing efficiency (reflected in increased effort or time) alongside modest impairment in performance effectiveness, particularly when task demands are high. Anxiety-related deficits in attention control have been observed across several behavioural measures. Trait anxious individuals, for example, perform less well on the executive attention subtest of the attention network task (ANT; Pacheco-Unguetti, Acosta, Callejas, \& Lupianez, 2010) and show deficits when required to inhibit task-irrelevant distractors or inhibit prepotent behavioural responses (review by Mobini \& Grant, 2007). In addition, studies have demonstrated the 


\section{PUPILLARY AND SACCADIC RESPONSES IN ANXIETY}

negative impact of elevated anxiety on processing efficiency in child (Hadwin, Brogan, \& Stevenson, 2005) and adult populations (see Eysenck et al., 2007).

Similarly, pupil diameter increases during periods of resource recruitment and mental effort (Beatty, 1982; Beatty \& Lucero-Wagoner, 2000; Hess, 1975; Kahneman \& Beatty, 1966; Karatekin, Marcus, \& Couperus, 2007). Trait anxious individuals show larger pupillary responses (vs. low anxious individuals) during tasks that require sustained attention (e.g. simulated driving tasks; Wilson, Smith, Chattington, Ford, and Marple-Horvat (2006), and pupillary responses are sensitive to anxiety-related deficits in other behavioural tasks such as learning paradigms (e.g. two-arm bandit learning task; Browning, Behrens, Jocham, O'Reilly, \& Bishop, 2015).

The antisaccade task is a simple behavioural task that utilises eye movement measures to investigate individual differences in attention control across anxiety and mood disorders (review by Ainsworth \& Garner, 2013). Participants are instructed to look away from a visual cue (i.e. to its mirror location) as quickly and accurately as possible. Attentional control is indicated in: (1) the ability to withhold (inhibit) reflexive saccades, and (2) the generation of volitional saccades to the correct location (Hutton \& Ettinger, 2006). Consistent with predictions from Attentional Control Theory (Eysenck et al., 2007), anxious individuals are more likely to make erroneous eye-movements on antisaccade trials (i.e. more likely to look towards the distractor stimulus), and are slower (less efficient) at executing antisaccades (Ansari \& Derakshan, 2011b; Garner, Attwood, Baldwin, James, \& Munafo, 2011). Furthermore, anxiety-related deficits in antisaccade performance are greater in response to threat distractors (e.g. angry facial expressions; Derakshan, Ansari, Hansard, Shoker, \& Eysenck, 2009; Reinholdt-Dunne et al., 2012) and when processing demands increase (Berggren, Richards, Taylor, \& Derakshan, 2013).

The effects of task demand/load have been examined in studies that manipulate the time between which a stimulus is presented and an eye-movement response is required. For example, participants make more errors and are slower to make accurate antisaccades when asked to respond immediately after stimulus onset (no-delay) compared to after a short delay, which is argued to facilitate response preparation (e.g. $600 \mathrm{~ms}-1500 \mathrm{~ms}$; Ansari \& Derakshan, 2010, 2011a; Reuter, Jager, Bottlender, \& Kathmann, 2007). Conversely, in an oculomotor delayed response (ODR) task a longer delay is introduced to increase demand on working memory. In this task, participants are required to encode the spatial location of a briefly presented visual cue and store information in working memory during a delay period (e.g. 5-10 secs), while maintaining a central fixation before generating a memory-guided saccade (Curtis \& D'Esposito, 2003; Luna \& Velanova, 2011). Consequently, the ODR task might be particularly sensitive to anxiety-related deficits in processing efficiency by increasing task demands in high load (long delay) conditions (Curtis \& D'Esposito, 2003). Furthermore, this task can incorporate concurrent online pupillometry measures that can profile the time-course of 'effort' expended throughout periods of high and low load (long and short delay respectively).

The current study asked whether individuals reporting elevated anxiety exhibit greater pupil reactivity to negative stimuli (threatening facial expressions) and subsequently have greater difficulty directing attention away from threat distractors, particularly when task demands are high. Specifically, we compared high and low anxious individuals' pupillary responses to centrally presented emotional (angry, fearful, happy, neutral) facial expressions and subsequent attention to faces presented in a memory-guided pro- and antisaccade ODR task under conditions of high and low cognitive load (following $10 \mathrm{~s}$ or $5 \mathrm{~s}$ delay respectively). We hypothesised that individuals with elevated levels of trait anxiety (trait anxious individuals) would show (1) larger pupillary responses to negative (angry, fearful) facial expressions (consistent with increased threat appraisal), (2) larger pupil responses during 


\section{PUPILLARY AND SACCADIC RESPONSES IN ANXIETY}

oculomotor delay, particularly during the long delay (consistent with greater effort/poor processing efficiency), and (3) impaired task performance characterised by fewer and slower accurate eye-movements, particularly on antisaccade trials in response to negative faces following a long delay (consistent with reduced ability to orient away from/inhibit threat distractors under conditions of high load).

\section{Method}

Participants

Participants were recruited from the University of Southampton via posters and an online advertisement. High and low anxious participants were selected according to their scores on the trait version of Spielberger State-Trait Anxiety Inventory (STAI-T; Spielberger, Gorusch, Lushene, Vagg, \& Jacobs, 1983). Consistent with previous research (see Ansari, Derakshan, $\&$ Richards, 2008) participants who scored $\leq 35$ were categorised as low anxious (LA; $n=$ 13 , mean STAI-T $=27.92$, S.D. $=4.11)$ and those scoring $\geq 50$ as high anxious $(\mathrm{HA} ; \mathrm{n}=14$, mean STAI-T $=56.79$, S.D. $=5.02$ ).

Participants had normal or corrected-to-normal vision and wore glasses or contact lenses if necessary. Participants confirmed that they had not taken drugs, alcohol, or medication on the day preceding testing. They received either course credits or $£ 6$ for participation. All participants provided informed consent. The research protocol was approved by the University of Southampton Ethics and Research Governance committees.

Apparatus

Eye movements and pupillary responses were recorded using SR Research EyeLink 1000 desktop-mounted eye tracking system (SR Research Ltd., Ontario, Canada) with a sampling rate of $1 \mathrm{kHz}$. Pupillary responses were recorded using Centroid model based on pupil diameter in millimetres. The presentation was controlled by Experiment Builder v1.10.1025 software (SR Research Ltd., Ontario, Canada) on a 19-inch ViewSonic (P227f) monitor. Stimuli

Sixty-four face stimuli were selected from the NimStim Face Stimulus Set (Tottenham et al., 2009) including angry, fearful, happy, and neutral expressions posed by 8 female and 8 male models (models were 02f, 03f, 05f, 06f, 07f, 08f, 09f, 18f, 24m, 25m, 26m, 28m, 30m, 33m, $34 \mathrm{~m}, 37 \mathrm{~m})$. Faces were standardized for size $\left(M_{\text {width }}=303 \mathrm{px}, M_{h e i g h t}=449 \mathrm{px}\right)$, aspect ratio (height/width; 1.48) and converted to grayscale with mean luminosity set to 116 . In order to adjust for head orientation and to centre the stimuli, the endocanthion (i.e. the point where the inner commissures of the lower and upper eyelid meet) line was rotated to become horizontal and the midpoint of this line was set to a stable coordinate of $253 \times 325$ pixels. In addition, a mask was applied to all face stimuli to cover hair and other external features and visual artefacts (e.g. scars, spots) were removed.

Experimental Task and Procedure

Each trial started with a drift correction phase. After participants fixated on the central fixation point, a face stimulus cue was presented in the centre of the screen for $1000 \mathrm{~ms}$, subtending $2^{\circ} 27^{\prime} \times 3^{\circ} 31^{\prime}$ of visual angle (viewing distance of $65 \mathrm{~cm}$ ). Following the presentation of the cue, the same face stimulus target (the eccentricity of each face from fixation cross $\left.=7^{\circ} 21^{\prime}\right)$ was presented at one of 8 compass points $\left(0^{\circ}, 45^{\circ}, 90^{\circ}, 135^{\circ}, 180^{\circ}\right.$, $\left.225^{\circ}, 270^{\circ}, 315^{\circ}\right)($ as per Ichihara-Takeda \& Funahashi, 2007; Park, 1997) for $150 \mathrm{~ms}$. After either a short (5 seconds) or long (10 seconds) delay, participants were instructed to look 'TOWARDS' (prosaccade) or 'AWAY' from (antisaccade) the location of the target (instruction $\mathrm{SOA}=300 \mathrm{~ms}$ ). The maximum time available to execute a pro- or antisaccade was $1500 \mathrm{~ms}$. A blank inter-trial interval (ITI) was presented for $1000 \mathrm{~ms}$. All stimuli were presented on a grey background $(\mathrm{R}=117, \mathrm{G}=116, \mathrm{~B}=116)$ and all trial screen displays had a mean luminosity of 116 . 


\section{PUPILLARY AND SACCADIC RESPONSES IN ANXIETY}

Participants were seated in front of the computer and eye-tracking system, with their chin and head rest at a viewing distance of $65 \mathrm{~cm}$. Participants were calibrated (9-point display) before completing 4 practice trials and the experimental task. The task consisted of 256 trials presented across 4 blocks of 64 trials (within each block 32 prosaccade and 32 antisaccade trials were presented in random order). Participants could take a small break between blocks and were re-calibrated when appropriate.

\section{Data Preparation}

Pupillary Responses. Pupillary responses were pre-processed using the following standard procedures (cf. Beatty \& Lucero-Wagoner, 2000; Bradley et al., 2008; Granholm, Morris, Asarnow, Chock, \& Jeste, 2000): epoching, blink-artefact removal and linear interpolation, baseline correction (relative to start of epoch), averaging, and selection of the peak pupil diameter by using MATLAB R2013a (The Math-Work, Inc., MA, USA). Peak pupillary responses (maximum pupil diameter) were calculated during each face cue (1000 ms), during the $5000 \mathrm{~ms}$ delay period (A), and during the first (B) and second (C) $5000 \mathrm{~ms}$ of the 10000 $\mathrm{ms}$ delay period. Responses during short delay $(\mathrm{A}+\mathrm{B} / 2)$ were compared against responses during the long delay $(\mathrm{C})$. Artefacts and blinks were defined as very short increases or decreases of at least $0.375 \mathrm{~mm}$ within $20 \mathrm{~ms}$ (Partala \& Surakka, 2003) and were removed. Missing values were corrected by linear interpolation resulting in $5.87 \%$ and $11.88 \%$ of data interpolation in face cue and delay periods respectively. Latency to peak pupillary response was measured for each face cue and delay type separately (i.e. the elapsed time between face/delay offset and peak pupil diameter).

Saccades. To assess task performance, mean latency (ms) and the percentage of saccadic error rates (\%) were calculated for pro- and antisaccades for each emotion and delay separately. Saccade onset was measured by subtracting the time of the first eye movement from the time of the instruction offset. Saccade accuracies and latencies (the first saccade following the offset of instruction) were extracted for the first valid saccade on each trial (i.e. latencies $>80 \mathrm{~ms}$, velocities $>30 \%$, and amplitudes $>3^{\circ}$ ) (Ansari \& Derakshan, 2010). This resulted in the loss of $8.56 \%$ of trials. An erroneous saccade was defined as the first saccade landing outside the relevant interest area for that trial type -i.e. $90^{\circ}$ quadrant around the face for prosaccade trials, and the $90^{\circ}$ quadrant in the opposite location for antisaccade trials. Statistical Analyses

Pupillary Responses. Amplitude and latency of peak pupil responses to face stimuli were entered into separate $2 \times 4$ repeated measures ANOVA with Group (low-anxious, highanxious) as the between subject factor and Emotion (angry, fearful, happy, neutral) as the within subject factor. Amplitude and latency of peak pupil responses to delay were entered into separate 2 × 2 × 4 repeated measures ANOVA with Group (low-anxious, high-anxious) as the between subject factor and Delay Type (short, long) and Emotion (angry, happy, fear, neutral) as the within subject factors.

Where assumptions of sphericity were violated, Greenhouse-Geisser corrections were applied. We conducted follow-up paired and independent samples t-tests where appropriate. Supplementary time-series analyses of pupil diameter examined effects of emotion and anxiety over-time across 50 consecutive $20 \mathrm{~ms}$ epochs as per Geva, Zivan, Warsha, and Olchik (2013).

Eye Movements. Saccade error (\%) and latency (ms) were analysed in separate 2 x 2 x 2 x 4 repeated measures ANOVAs with Group (low-anxious, high-anxious) as the between subject factor and Saccade Type (antisaccade, prosaccade), Delay Type (short, long) and Emotion (angry, fearful, happy, neutral) as the within subject factors. As before, Greenhouse-Geisser 


\section{PUPILLARY AND SACCADIC RESPONSES IN ANXIETY}

corrections were applied where sphericity was violated, and we used paired and independent samples t-tests to explore interactions where appropriate.

\section{Results}

\section{Pupillary Responses}

Emotion facial expressions. Peak pupillary responses to facial expressions were larger but slower in high-anxious relative to low-anxious individuals, (Amplitude: High-anxious $(M=$ $0.16, S . D .=0.12)$ vs low-anxious $(M=0.10, S . D .=0.05), F(1,21)=5.21, p=.03, \eta^{2}=.19$; Latency: High-anxious $(M=239.42, S . D .=89.79)$ vs low-anxious $(M=172.12, S . D .=75.89$ ), $\left.F(1,21)=4.44, p=.04, \eta^{2}=.17\right)$. The time series analyses suggested that differences in pupil dilation between high and low anxiety groups were most pronounced from 300-400 ms after stimulus onset (see Figure 1).

A main effect of emotion $\left[F(3,63)=6.08, p=.001, \eta^{2}=.22\right]$ was characterised by larger peak responses to angry $(M=0.17, S . D .=0.12)$ relative to happy $(M=0.10, S . D .=0.09)$

expressions - time-series analysis suggests this effect was most pronounced from 200-300 ms (Figure 2). There was no effect of anxiety $\mathrm{x}$ emotion on peak response, nor latency (Fs $<1 \mathrm{ps}$ $>.1)$.

Short and long delays. A Group x Delay interaction $\left[F(1,22)=4.68, p=.04, \eta^{2}=.17\right]$ was characterised by smaller peak pupil amplitude in low anxious individuals during short delay compared to i) long delay in low anxious individuals $(t(13)=2.97, p=.006)$, and ii) peak responses in high anxious individuals during long delay $(t(13)=2.06, p=.04)$ and short delay $(t(13)=2.67, p=.01)$ (see Figure 3). Peak amplitudes in high anxious individuals were unaffected by delay, and were comparable to the elevated peak amplitude in low anxious individuals during long delay. Comparable analyses of peak latency did not reveal significant effects of group, delay, emotion, nor their interaction, Fs $<1 \mathrm{ps}>$.1. Supplementary timeseries analyses suggested the difference between short and long delay in low anxious individuals reflects an increase in pupil diameter towards the end of the long delay period ( $>$ $8500 \mathrm{~ms}$,) (see Figure 4). Consistent with peak analyses, time-series analysis did not reveal significant effects in the high anxious participants.

\section{Eye-movement saccade error and latency}

Participants made more errors $(\%)$ on antisaccade trials $(M=19.43, S . D .=11.41)$ than prosaccade trials $(M=16.28, S . D .=8.94),\left[F(1,25)=4.09, p=.05, \eta^{2}=.14\right]$, irrespective of delay, emotion and anxiety group, $\mathrm{Fs}<1 \mathrm{ps}>$.1. A significant anxiety Group x Delay interaction $\left[F(1,25)=9.22, p=.001, \eta^{2}=.26\right]$ was characterised by an increased number of eye-movement errors in high (vs. low) anxious individuals following long delays $t(13)=3.28$, $p=.005$ ) (see Figure 5). A corresponding analysis of eye-movement latencies indicated that participants were slower to make correct saccades on antisaccade $(M=246.67, S . D .=60.30)$ vs. prosaccade trials $(M=233.66, S . D .=52.54),\left[F(1,25)=4.77, p=.03, \eta^{2}=.16\right]$, and following short delay $(M=250.39, S . D .=52.32)$ compared to long delay $(M=229.94, S . D .=$ 58.76), $\left[F(1,25)=19.91, p=.05, \eta^{2}=.44\right]$.

\section{Discussion}

We examined pupillometric and saccadic measures of affective and attentional processing in high and low anxious groups in an ODR task. High (vs low) anxious individuals produced larger and slower (sustained) pupillary responses to face stimuli, larger pupillary responses during the short delay and more erroneous eye-movements (across pro and antisaccade trials), particularly following long delay. Across groups, pupillary responses were larger to angry compared to happy faces. 


\section{PUPILLARY AND SACCADIC RESPONSES IN ANXIETY}

Evidence of large and sustained pupil responses to face stimuli in anxiety extends evidence of pupil increases to angry faces in anxious individuals (Kret et al., 2013) and children of anxious mothers (Burkhouse et al., 2014). Contrary to predictions, anxiety was not characterised by larger pupil responses to negative (angry or fearful) facial expressions. Rather, across participants there was evidence of larger pupil responses to angry faces, consistent with previous studies in unselected samples (Bradley et al., 2008; Partala \& Surakka, 2003; Rosa et al., 2015). Pupil responses were not greater to other negative faces in our study (i.e. fear faces), consistent with see Kret et al. (2013), but contrary to the effects of fear faces on other autonomic measures (heart-rate, skin conductance) and neural activity in brain regions implicated in threat appraisal (i.e. the amygdala, Adolphs, Tranel, Damasio, \& Damasio, 1995; Öhman, 2005). Previous studies have either not examined or not found strong effects of fear faces on pupil diameter, and future studies are required to directly compare pupil response to a range of emotional expressions and other stimuli (e.g., emotional sounds and images) to determine the specificity of pupil responses to negative or broadly emotional stimuli in anxiety.

In the current study, anxiety-group differences were most pronounced between 300-400 ms after the face onset and were slower to return to baseline, consistent with relatively early effects of appraisal and attention to face stimuli in anxiety (see the review by Cisler \& Koster, 2010). Sustained pupil responses in anxious individuals have been observed in other face-viewing paradigms. Price et al. (2013) observed large and sustained pupil responses 2-10 seconds after attention had been cued to the location of fear faces in a visual probe task. The authors argued that this effect reflected inflexible pupil reactivity during sustained cognitiveaffective load (Price et al., 2013; Figure 2A). Interestingly, we observed similarly large pupil responses in anxious individuals during both 5 and 10 second delay periods, whereas low anxious individuals exhibited smaller pupil responses during the short compared to the long delay. This result might reflect increased effort in anxious individuals during short delay alongside low anxious individuals' capacity to reduce effort in low-load/short-delay trials, consistent with flexible resource allocation in low-anxious individuals. Convergent evidence comes from learning paradigms (e.g. two-arm bandit learning task with stable and unstable shock) in which low anxious individuals' pupil responses are more sensitive to changes in environmental contingencies across stable vs. unstable (i.e. volatile) blocks in learning paradigms (Browning et al., 2015).

Together these findings reflect sustained but inflexible patterns of pupil responding during affective stimulus processing and cognitive load in anxious individuals that contrast with adaptive responding to task demands in low anxious individuals. As noted by others, elevated, sustained and inflexible pupil responding in anxiety might reflect individual differences in central arousal mechanisms including the locus-coeruleus-norepinephrine (LCNE) system (Browning et al., 2015). For example, the present findings might indicate elevations in tonic LC-NE activity in high trait anxious individuals (Howells, Stein, \& Russell, 2012) that increase and sustain phasic responses to salient stimuli (i.e. centrally presented face stimuli in our study). Phasic responses may then become more inflexible and resistant to affective/cognitive processing as anxiety or task demands increase.

High trait anxious individuals made more eye-movement errors across prosaccade and antisaccade trials, particularly following a long delay period that in the current task increased working memory load. This general deficit in performance accuracy (effectiveness) was not increased in response to threat faces, nor was it linked to impaired processing efficiency, that is, latencies to make accurate saccades were unaffected by anxiety. This might reflect several features of the task used; notably (1) the affective salience of threat faces may have reduced following their repeated presentation within each trial (first at central fixation and then in the periphery) (see Van Dam, Earleywine, \& Altarriba, 2012) and (2) the delay period may have 


\section{PUPILLARY AND SACCADIC RESPONSES IN ANXIETY}

further reduced the affective salience of face cues and placed sufficient demands on working memory (see Berggren et al., 2013) to elicit pronounced anxiety-related deficits in performance effectiveness/accuracy rather than on efficiency. Our antisaccade error rates and latencies were similar to those observed in other studies in healthy (Taylor \& Hutton, 2009, 2011) and preselected high and low trait anxious individuals (Ansari \& Derakshan, 2010). However we did observe comparatively high prosaccade error rates and slower prosaccades across participants. This likely reflects an added cost on prosaccades when participants wait for prolonged periods to execute saccades to one of eight discrete target regions of interest. Our findings suggest that pupil diameter can provide a comparatively simple and objective measure of dynamic changes in emotion processing and cognitive effort in anxiety, to extend studies of subjective effort (Hadwin et al., 2005). Evidence of increased and sustained pupil responses in trait anxious individuals across experimental conditions of low and high cognitive load warrants replication in larger samples and clinical groups (e.g. generalized anxiety disorder). To this end future pupillometry studies could be used in experimental designs that can dissociate individual differences in early appraisal mechanisms, refractory and preparatory behavioural responses in complex cognitive tasks. For example, in addition to providing a convergent measure of early autonomic responses to emotional cues (e.g., skin conductance), pupillometry provides a non-intrusive online measure of individual differences in temporal changes in task un-related processes that underlie perseverative patterns of negative thinking e.g. worry in anxiety and rumination in depression.

\section{$<$ REF $>$ References}

$<$ BIBL $>$

Adolphs, R., Tranel, D., Damasio, H., \& Damasio, A. R.;1; (1995). Fear and the human amygdala. $J$ Neurosci, 15(9), 5879-5891. <DOI $>10.1093 /$ neucas/3.4.267</DOI $>$

Ainsworth, B., \& Garner, M.;1; (2013). Attention control in mood and anxiety disorders: evidence from the antisaccade task. Hum Psychopharmacol, 28(3), 274-280.

$<$ DOI $>10.1002 /$ hup. $2320<$ DOI $>$

Andreassi, J. L.;1; (2000). Pupillary response and behavior Psychophysiology: Human Behavior \& Physiological Response (4 ed., pp. 218-233). $<$ PL $>$ New Jersey $</$ PL $>$ : Lawrance Erlbaum Assoc.

Ansari, T. L., \& Derakshan, N.;1; (2010). Anxiety impairs inhibitory control but not volitional action control. Cogn Emot, 24(2), 241-254.

$<$ DOI $>10.1080 / 02699930903381531</$ DOI $>$

Ansari, T. L., \& Derakshan, N.;1; (2011a). The neural correlates of cognitive effort in anxiety: effects on processing efficiency. Biol Psychol, 86(3), 337-348.

$<$ DOI $>10.1016 /$ j.biopsycho.2010.12.013</DOI $>$

Ansari, T. L., \& Derakshan, N.;1; (2011b). The neural correlates of impaired inhibitory control in anxiety. Neuropsychologia, 49(5), 1146-1153.

$<$ DOI $>10.1016 /$ j.neuropsychologia.2011.01.019</DOI $>$

Ansari, T. L., Derakshan, N., \& Richards, A.;1; (2008). Effects of anxiety on task switching: Evidence from the mixed antisaccade task. Cognitive, Affective, \& Behavioral Neuroscience, 8(3), 229-238. <DOI $>10.3758 /$ cabn.8.3.229</DOI $>$ 


\section{PUPILLARY AND SACCADIC RESPONSES IN ANXIETY}

Bar-Haim, Y., Lamy, D., Pergamin, L., Bakermans-Kranenburg, M. J., \& van IJzendoorn, M. H.;1; (2007). Threat-related attentional bias in anxious and nonanxious individuals: a metaanalytic study. Psychol Bull, 133(1), 1-24. <DOI $>10.1037 / 0033-2909.133 .1 .1</$ DOI $>$

Beatty, J.;1; (1982). Task-evoked pupillary responses, processing load, and the structure of processing resources. Psychol Bull, 91(2), 276-292. <DOI $>10.1037 / / 0033-$ 2909.91.2.276</DOI $>$

Beatty, J., \& Lucero-Wagoner, B.;1; (2000). The Pupillary System. In J. T. Cacioppo, L. G. Tassinary, \& G. G. Bernston (Eds.), Handbook of Psychophysiology (pp. 142-162). $<$ PL $>$ NY: Cambridge $</ \mathrm{PL}><\mathrm{PN}>$ University Press $</ \mathrm{PN}>$.

Behar, E., DiMarco, I. D., Hekler, E. B., Mohlman, J., \& Staples, A. M.;1; (2009). Current theoretical models of generalized anxiety disorder (GAD): Conceptual review and treatment implications. J Anxiety Disord, 23(8), 1011-1023.

$<$ DOI $>10.1016 /$ j.janxdis.2009.07.006</DOI $>$

Berggren, N., Richards, A., Taylor, J., \& Derakshan, N.;1; (2013). Affective attention under cognitive load: reduced emotional biases but emergent anxiety-related costs to inhibitory control. Front Hum Neurosci, 7, 188. <DOI>10.3389/fnhum.2013.00188</DOI >

Bishop, S. J.;1; (2009). Trait anxiety and impoverished prefrontal control of attention. Nat Neurosci, 12(1), 92-98. <DOI $>10.1038 / \mathrm{nn} .2242</$ DOI $>$

Bradley, M. M., Miccoli, L., Escrig, M. A., \& Lang, P. J.;1; (2008). The pupil as a measure of emotional arousal and autonomic activation. Psychophysiology, 45(4), 602-607.

$<$ DOI $>10.1111 /$ j.1469-8986.2008.00654. $\mathrm{x}<$ /DOI $>$

Browning, M., Behrens, T. E., Jocham, G., O'Reilly, J. X., \& Bishop, S. J.;1; (2015). Anxious individuals have difficulty learning the causal statistics of aversive environments. Nat Neurosci, 18(4), 590-596. <DOI >10.1038/nn.3961</DOI $>$

Burkhouse, K. L., Siegle, G. J., \& Gibb, B. E.;1; (2014). Pupillary reactivity to emotional stimuli in children of depressed and anxious mothers. J Child Psychol Psychiatry, 55(9), 1009-1016. <DOI $>10.1111 /$ jcpp. $12225</$ DOI $>$

Chen, N. T., Clarke, P. J., Watson, T. L., Macleod, C., \& Guastella, A. J.;1; (2014). Biased saccadic responses to emotional stimuli in anxiety: an antisaccade study. PLoS One, 9(2), e86474. $<$ DOI $>10.1371 /$ journal.pone.0086474</DOI $>$

Cisler, J. M., \& Koster, E. H.;1; (2010). Mechanisms of attentional biases towards threat in anxiety disorders: An integrative review. Clin Psychol Rev, 30(2), 203-216.

$<$ DOI $>10.1016 /$ j.cpr.2009.11.003 $<$ /DOI $>$

Curtis, C. E., \& D'Esposito, M.;1; (2003). Persistent activity in the prefrontal cortex during working memory. Trends Cogn Sci, 7(9), 415-423. <DOI>10.1016/s1364-6613(03)00197$9</ \mathrm{DOI}>$

Derakshan, N., Ansari, T. L., Hansard, M., Shoker, L., \& Eysenck, M. W.;1; (2009). Anxiety, inhibition, efficiency, and effectiveness. An investigation using antisaccade task. Exp

Psychol, 56(1), 48-55. <DOI $>10.1027 / 1618-3169.56 .1 .48</$ DOI $>$ 


\section{PUPILLARY AND SACCADIC RESPONSES IN ANXIETY}

Derryberry, D., \& Reed, M. A.;1; (2002). Anxiety-related attentional biases and their regulation by attentional control. J Abnorm Psychol, 111(2), 225-236. <DOI >10.1037//0021$843 \mathrm{X} .111 .2 .225</ \mathrm{DOI}>$

Eysenck, M. W., \& Calvo, M. G.;1; (1992). Anxiety and Performance: The Processing Efficiency Theory. Cogn Emot, 6(6), 409-434. <DOI>10.1080/02699939208409696</DOI>

Eysenck, M. W., \& Derakshan, N.;1; (2011). New perspectives in attentional control theory. Personality and Individual Differences, 50(7), 955-960.

$<$ DOI $>10.1016 /$ j.paid.2010.08.019</DOI $>$

Eysenck, M. W., Derakshan, N., Santos, R., \& Calvo, M. G.;1; (2007). Anxiety and cognitive performance: attentional control theory. Emotion, 7(2), 336-353. $<$ DOI $>10.1037 / 1528-$ 3542.7.2.336</DOI $>$

Garner, M., Attwood, A., Baldwin, D. S., James, A., \& Munafo, M. R.;1; (2011). Inhalation of $7.5 \%$ carbon dioxide increases threat processing in humans. Neuropsychopharmacology, 36(8), 1557-1562. <DOI $>10.1038 /$ npp.2011.15</DOI $>$

Geva, R., Zivan, M., Warsha, A., \& Olchik, D.;1; (2013). Alerting, orienting or executive attention networks: differential patters of pupil dilations. Front Behav Neurosci, 7, 145. $<$ DOI $>10.3389 /$ fnbeh.2013.00145 $<$ /DOI $>$

Granholm, E., Morris, S., Asarnow, R. F., Chock, D., \& Jeste, D. V.;1; (2000). Accelerated age-related decline in processing resources in schizophrenia: evidence from pupillary responses recorded during the span of apprehension task. J Int Neuropsychol Soc, 6(1), 3043. $<$ DOI $>10.1017 / \mathrm{s} 1355617700611049</$ DOI $>$

Grupe, D. W., \& Nitschke, J. B.;1; (2013). Uncertainty and anticipation in anxiety: an integrated neurobiological and psychological perspective. Nat Rev Neurosci, 14(7), 488-501. $<$ DOI $>10.1038 / \mathrm{nrn} 3524</$ DOI $>$

Hadwin, J. A., Brogan, J., \& Stevenson, J.;1; (2005). State anxiety and working memory in children: A test of processing efficiency theory. Educational Psychology, 25(4), 379-393. $<$ DOI $>10.1080 / 01443410500041607</$ DOI $>$

Hess, E. H.;1; (1975). The Tell-Tale Eye: How Your Eyes Reveal Hidden Thoughts and Emotions. $<\mathrm{PL}>\mathrm{NY}<$ PL $>$ : van Nostrand Reinhold.

Howells, F. M., Stein, D. J., \& Russell, V. A.;1; (2012). Synergistic tonic and phasic activity of the locus coeruleus norepinephrine (LC-NE) arousal system is required for optimal attentional performance. Metab Brain Dis, 27(3), 267-274. <DOI>10.1007/s11011-012-9287$9</ \mathrm{DOI}>$

Hutton, S. B., \& Ettinger, U.;1; (2006). The antisaccade task as a research tool in psychopathology: a critical review. Psychophysiology, 43(3), 302-313.

$<$ DOI $>10.1111 /$ j.1469-8986.2006.00403. $\mathrm{x}<$ /DOI $>$ 


\section{PUPILLARY AND SACCADIC RESPONSES IN ANXIETY}

Ichihara-Takeda, S., \& Funahashi, S.;1; (2007). Activity of primate orbitofrontal and dorsolateral prefrontal neurons: task-related activity during an oculomotor delayed-response task. Exp Brain Res, 181(3), 409-425. <DOI>10.1007/s00221-007-0941-0</DOI >

Kahneman, D., \& Beatty, J.;1; (1966). Pupil diameter and load on memory. Science, 154(3756), 1583-1585. <DOI $>10.1126 /$ science.154.3756.1583</DOI $>$

Karatekin, C., Marcus, D. J., \& Couperus, J. W.;1; (2007). Regulation of cognitive resources during sustained attention and working memory in 10-year-olds and adults.

Psychophysiology, 44(1), 128-144. $<$ DOI $>10.1111 / \mathrm{j} .1469-8986.2006 .00477 . \mathrm{x}</ \mathrm{DOI}>$

Kret, M. E., Stekelenburg, J. J., Roelofs, K., \& de Gelder, B.;1; (2013). Perception of face and body expressions using electromyography, pupillometry and gaze measures. Front Psychol, 4, 28. <DOI $>10.3389 /$ fpsyg.2013.00028</DOI $>$

Luna, B., \& Velanova, K.;1; (2011). Development from reflexive to controlled eye movements. In S. P. Liversedge, I. D. Gilchrist, \& S. Everling (Eds.), The Oxford Handbook of Eye Movements (pp. 621-642). $<\mathrm{PL}>\mathrm{NY}$ : Oxford $</ \mathrm{PL}><\mathrm{PN}>$ University Press $</ \mathrm{PN}>$. Mobini, S., \& Grant, A.;1; (2007). Clinical implications of attentional bias in anxiety disorders: An integrative literature review. Psychotherapy (Chic), 44(4), 450-462. $<$ DOI $>10.1037 / 0033-3204.44 .4 .450</$ DOI $>$

Mogg, K., \& Bradley, B. P.;1; (2016). Anxiety and attention to threat: Cognitive mechanisms and treatment with attention bias modification. Behav Res Ther, 87, 76-108.

$<$ DOI $>10.1016 /$ j.brat.2016.08.001 $<$ DOI $>$

Mogg, K., Garner, M., \& Bradley, B. P.;1; (2007). Anxiety and orienting of gaze to angry and fearful faces. Biol Psychol, 76(3), 163-169. <DOI>10.1016/j.biopsycho.2007.07.005</DOI >

Öhman, A.; 1 ; (2005). The role of the amygdala in human fear: automatic detection of threat. Psychoneuroendocrinology, 30(10), 953-958.

$<$ DOI $>10.1016 /$ j.psyneuen.2005.03.019</DOI $>$

Pacheco-Unguetti, A. P., Acosta, A., Callejas, A., \& Lupianez, J.;1; (2010). Attention and anxiety: different attentional functioning under state and trait anxiety. Psychol Sci, 21(2), 298-304. $<$ DOI $>10.1177 / 0956797609359624</$ DOI $>$

Park, S.;1; (1997). Association of an oculomotor delayed response task and the Wisconsin Card Sort Test in schizophrenic patients. International Journal of Psychophysiology, 27, 147151.

Partala, T., \& Surakka, V.;1; (2003). Pupil size variation as an indication of affective processing. International Journal of Human-Computer Studies, 59(1-2), 185-198. $<$ DOI $>10.1016 /$ S1071-5819(03)00017-X</DOI $>$

Price, R. B., Siegle, G. J., Silk, J. S., Ladouceur, C., McFarland, A., Dahl, R. E., \& Ryan, N. D.;1; (2013). Sustained neural alterations in anxious youth performing an attentional bias task: a pupilometry study. Depress Anxiety, 30(1), 22-30. $<$ DOI $>10.1002 /$ da.21966</DOI $>$

Reinholdt-Dunne, M. L., Mogg, K., Benson, V., Bradley, B. P., Hardin, M. G., Liversedge, S. P.,;1;... Ernst, M. (2012). Anxiety and selective attention to angry faces: An antisaccade 


\section{PUPILLARY AND SACCADIC RESPONSES IN ANXIETY}

study. Journal of Cognitive Psychology, 24(1), 54-65.

$<$ DOI $>10.1080 / 20445911.2011 .560111<$ DOI $>$

Reuter, B., Jager, M., Bottlender, R., \& Kathmann, N.;1; (2007). Impaired action control in schizophrenia: the role of volitional saccade initiation. Neuropsychologia, 45(8), 1840-1848. $<$ DOI $>10.1016 /$ j.neuropsychologia.2006.12.006</DOI $>$

Richards, H. J., Benson, V., Donnelly, N., \& Hadwin, J. A.;1; (2014). Exploring the function of selective attention and hypervigilance for threat in anxiety. Clin Psychol Rev, 34(1), 1-13. $<$ DOI $>10.1016 /$ j.cpr.2013.10.006 </DOI $>$

Rosa, P. J., Esteves, F., \& Arriaga, P.;1; (2015). Beyond Traditional Clinical Measurements for Screening Fears and Phobias. IEEE Transactions on Instrumentation and Measurement, 64(12), 3396-3404. <DOI $>10.1109 /$ tim.2015.2450292</DOI $>$

Spielberger, C. D., Gorusch, R. L., Lushene, R., Vagg, P. R., \& Jacobs, G. A.;1; (1983). Manual for State-Trait Anxiety Inventory. $<\mathrm{PL}>$ Palo Alto, $\mathrm{CA}</ \mathrm{PL}>$ : Consulting Psychologists Press.

Sylvester, C. M., Corbetta, M., Raichle, M. E., Rodebaugh, T. L., Schlaggar, B. L., Sheline, Y. I.,;1;. .. Lenze, E. J. (2012). Functional network dysfunction in anxiety and anxiety disorders. Trends Neurosci, 35(9), 527-535. <DOI >10.1016/j.tins.2012.04.012</DOI $>$

Taylor, A. J., \& Hutton, S. B.;1; (2009). The effects of task instructions on pro and antisaccade performance. Exp Brain Res, 195(1), 5-14. <DOI >10.1007/s00221-009-1750$4</$ DOI $>$

Taylor, A. J., \& Hutton, S. B.;1; (2011). Error awareness and antisaccade performance. Exp Brain Res, 213(1), 27-34. <DOI $>10.1007 / \mathrm{s} 00221-011-2770-4</ \mathrm{DOI}>$

Tottenham, N., Tanaka, J. W., Leon, A. C., McCarry, T., Nurse, M., Hare, T. A.,;1;... Nelson, C. (2009). The NimStim set of facial expressions: judgments from untrained research participants. Psychiatry Res, 168(3), 242-249. <DOI >10.1016/j.psychres.2008.05.006</DOI $>$

Van Dam, N. T., Earleywine, M., \& Altarriba, J.;1; (2012). Anxiety attenuates awareness of emotional faces during rapid serial visual presentation. Emotion, 12(4), 796-806.

$<$ DOI $>10.1037 / \mathrm{a} 0024648</$ DOI $>$

Wilson, M., Smith, N. C., Chattington, M., Ford, M., \& Marple-Horvat, D. E.;1; (2006). The role of effort in moderating the anxiety-performance relationship: Testing the prediction of processing efficiency theory in simulated rally driving. $J$ Sports $S c i, 24(11), 1223-1233$. $<$ DOI $>10.1080 / 02640410500497667</$ DOI $>$

$</ \mathrm{BIBL}>$

$<$ Figure $>$ Figure 1. Pupillary response time series to face cues in high-anxious (HA) and lowanxious (LA) groups (bars represent standard errors). 


\section{PUPILLARY AND SACCADIC RESPONSES IN ANXIETY}

$<$ Figure $>$ Figure 2. Pupillary response time series to face cues (bars represent standard errors).

$<$ Figure $>$ Figure 3. Peak pupillary responses during long and short delay trials in highanxious (HA) and low-anxious (LA) groups (bars represent standard errors

$<$ Figure $>$ Figure 4. Pupillary response time series for long and short delay trials in highanxious (HA) and low-anxious (LA) groups (bars represent standard errors) (Upper and lower $\mathrm{x}$-axes represent time points during short and long delay, respectively).

$<$ Figure $>$ Figure 5. Percentage of saccade error in high-anxious (HA) and low-anxious (LA) groups on each of the long and short delay trials (bars represent standard errors).

TDENDOFDOCTD 